\title{
Trust Requirements Model for Developing Acceptable Autonomous Car
}

\author{
Halimaton Hakimi $^{1}$, Massila Kamalrudin ${ }^{2}$, Safiah Sidek $^{3}$, Suriati Akmal ${ }^{2}$ \\ ${ }^{1}$ Faculty of Information and Communication Technology, Universiti Teknikal Malaysia Melaka, Melaka, Malaysia \\ ${ }^{2}$ Innovative Software System and Services Group, Universiti Teknikal Malaysia, Melaka, Malaysia \\ ${ }^{3}$ Institute of Technology Management and Entrepreneurship, Universiti Teknikal Malaysia Melaka, Melaka, Malaysia
}

\section{Email address:}

halimaton.mpp1516@gmail.com (H. Hakimi),massila@utem.edu.my (M. Kamalrudin), safiahsidek@utem.edu.my (S. Sidek), suriatiakmal@utem.edu.my (S. Akmal)

\section{To cite this article:}

Halimaton Hakimi, Massila Kamalrudin, Safiah Sidek, Suriati Akmal. Trust Requirements Model for Developing Acceptable Autonomous Car. Journal of Electrical and Electronic Engineering. Vol. 6, No. 2, 2018, pp. 59-64. doi: 10.11648/j.jeee.20180602.14

Received: May 2, 2018; Accepted: June 6, 2018; Published: June 20, 2018

\begin{abstract}
There has been an increase interest among automakers to develop autonomous cars. However, the level of acceptance of the autonomous cars among users is limited. Considering that trust is one of the main determinants for users to accept the autonomous cars. Considering that user's needs and expectation are highly important when developing the autonomous car, a trust requirements model that consists of attributes and related properties based on the perspectives of the users has been developed. The model was also developed based on the proposition that automakers need to consider trust requirements at the early stage of developing the autonomous car. Drawn from a systematic analysis of the literature review, seven attributes, namely safety, security, privacy, performance, user's experience, reliability and economic value together with their related properties were identified. It was also found that there is a one-to-many relationship between the attribute and its properties. This model, named as trust requirements autonomous car (TReAC) model can be used as guideline for automakers to develop acceptable autonomous cars. It is anticipated that this model can be adaptable to other domain. Future work should be dedicated to validating and testing this model.
\end{abstract}

Keywords: Autonomous Car, Trust Requirements, TReAC Model, User Acceptable

\section{Introduction}

The automotive industry has been experiencing a new revolution in the automobile technology, that is the development of autonomous cars, referred as the driverless cars. This development is also in line with the Mega Science 3.0 Roadmap 2020-2050 that requires the industry to provide a safer and more efficient driver-free driving. Autonomous cars (ACs) are driverless cars that incorporate a control function without direct driver input. The National Highway Traffic Safety Administration (NHTSA, 2016) describes that the autonomous cars have some aspects of safety-critical control function, such as steering, brake assist, self-parking and other functions without a direct driver input [1]. Although driving ACs is argued to greatly improve safety by reducing collisions resulting from human error and human distraction [2], the acceptance level of autonomous cars is still minimal due to the lack of trust on the autonomous technology by the users. In this case, trust requirements need to be related to user's confidence of the autonomous car, which is derived from the provider's efforts in considering the safety, security, privacy and performance that meet the user's expectations. This is because accurate definition of trust requirements helps users to choose and use AC that have high level of safety as well as to prevent users from getting involved in accidents.

Emphasizing the need to reduce $94 \%$ of car collisions due to human error, the development of autonomous is experiencing a rapid growth parallel with the demand on the revolution Industry 4.0. However, most of the users do not believe the ACs can address security and safety [ref]. In this regard, the acceptance level of autonomous car is still minimal due to the lack of trust on the autonomous technology.

Further, lack of trust is one of the impacts that reduces 
user's acceptance on the ACs. Acceptance is a post-hoc concept and generally measured after exposure, whereby users can express their support either via attitude or behavioral response. The majority of users could not accept a car that is not controlled manually by human, when needed. This is a clear indication that user acceptance is the main barrier for user's acceptance on the technology of autonomous car or full automation [2]. In reality, everybody wants their own car to protect them at all cost. This aspiration is consistent with the development of autonomous cars that address the issues of "safety dilemma" for the purpose of reducing the number of casualties. It is expected that the development of autonomous car will eventually reduce the rates of collision, leading to safety improvement. In this case, the development of autonomous car needs to consider the issues of trust and user acceptance.

Recently, [4] focus on the human-car relationship in analyzing trust issue. They proposed a framework for integrating mutual trust computation with a standard humanrobot interaction. As a result, the framework of trust allows the connection of multi-modal sensor data from the autonomous vehicle and data from user's handheld device. Although this framework leverages the trust related to human-car interaction through Human-Robot interaction platform, it lacks the properties of trust between human and machine. Further, it also deemphasizes the needs for manufacturers to develop autonomous cars that are compatible to user acceptance. As such, this paper proposes a new trust model that addresses user acceptance on autonomous car.

The rest of this paper is organized as follows: Section II presents the background and motivation. Section III presents the trust requirements model proposed in this paper. Section IV concludes the paper with some discussions about trust model and future works.

\section{Background and Motivation}

\subsection{Definition of Requirements}

Requirements are the needs or expectations of customers regarding the product developed in a particular industry. In this case, the product developed by the industry is the autonomous car. There are two main reasons why requirements are necessary for a particular product, which are: i) Specific product demands certain functions or qualities, and ii) customer wants that requirements to be part of the development of the product [5]. Therefore, requirements based on user's needs are essential for the creation and development of the autonomous car.

Requirements consists of two classifications: functional requirements and non-functional requirements. Functional requirements is an action that the product must take if it is to be useful to its user. It relates to any actions, such as calculation, technical details or other specific functionality that define what a system is supposed to accomplish.
Non-functional requirements specifies how the product should work and the qualities the product must have. The non-functional requirements for autonomous car describes properties, such as the look and feel of safety, security and privacy, which are critical to the product's success based on the user's expectations and demand. In this case, correct nonfunctional requirements are important to ensure the developed autonomous car look secure and safe.

In the next section, we describe the trust requirement.

\subsection{Trust Requirements}

Trust requirements are defined as the needs to address the issues of safety, reliability and comfort of drivers or users [6]. The trust preference is based on three aspects: i) Customer's satisfaction with the technology provided, ii) Willingness of learning and using the automated features in the current autonomous technology in a car, and iii) Preferable method for learning to use the automated features [7].

The importance of trust in the autonomous car is related to the factors for creating willingness to use an autonomous car system and ensuring its correct usage [8]. Currently, most studies focus on user's expectations or acceptance of the system, ignoring the trust aspect of the autonomous system. It is identified that trust plays an important role in enhancing the acceptance of autonomous cars. Trust contributes to the confidence level of the drivers, especially when they achieve knowledge-drive and emotion drive [9].

In summary, it is important to develop an autonomous car based on user's need and expectations, including the trust factors that are able to enhance the acceptance of the autonomous technology. Yet, most of the existing works [8] [10] [11] tend to focus only on human-machine interaction rather than user acceptance. Thus, there is a need to propose trust requirements model that addresses user acceptance on the autonomous car, named as the Trust Requirements Autonomous Car (TReAC) model.

\subsection{Levels of Autonomous Driving}

The different levels of autonomous driving, as shown in Table 1 serve as a general guideline for users determine the level of automation of vehicles [12]. This guideline has been used as reference by many projects related to the development of autonomous cars [13], although some research indicated that level 5 has not been implemented on British roads until 2030 due the lack of trust among drivers on autonomous cars $[14,15]$.

Semi-autonomous functions as example level 3 and level 4 in relatively formulaic situation such as parking and cruise control already available, and a fully autonomous car is expected to be introduced in the near future. According to table 1 , there is a huge difference of function from level 1 to 3 , where starting from level 3, semi and fully automated functions are introduced in the autonomous cars. This indicates that higher trust is required starting from level 3 to 5 . 
Table 1. The Five Levels of Autonomous Driving [NHTSA, 2016]

\begin{tabular}{ll}
\hline Level 0 & Zero Automation: Driving as Usual \\
& Function: A human driver is required to operate the vehicle safely at all times. \\
Driver Assisted/Function Specific: & Intelligent Features add layer of safety and comfort \\
Level 1 & Function: A human driver required for all critical function. The car can alert the driver to condition, environment and obstructions. It can also \\
& offer assisted/smart performance and driving capabilities. \\
Partial Automation/Combined Autonomous Functions: & Key Automated capabilities become standard but driver still in control \\
Level 2 & $\begin{array}{l}\text { Function: A least two simultaneous autonomous tasks become are managed by the vehicle in specific scenario. } \\
\text { Conditional Automation/Limited Self Driving: The car becomes a co-pilot }\end{array}$ \\
Level 3 & $\begin{array}{l}\text { Function: The vehicle manages most safety-critical driving functions as known (mapped) environmental conditions. A human driver is still } \\
\text { present and expected to manage vehicle operation. }\end{array}$ \\
High Automation: Capable of performing all safety-critical driving functions while monitoring environment/conditions in defined use cases \\
Function: Per NHTSA, this is full self-driving automation. Per SAE, self-driving is fully possible in most road conditions and environments \\
without need of human intervention. A functional driver cockpit is still in place (steering wheel, brake/acceleration pedal). \\
Fully Autonomous: Vehicle is completely Driverless
\end{tabular}

\section{Trust Requirements Model for Autonomous Car}

Figure 1 shows the trust requirement model for developing autonomous car (TReAC). The model is developed based on the literature study. The model consists of trust attribute and its related trust properties. The development of the model starts with the determination of the attributes of trust related to the usage of autonomous car. Each attributes is further analyse with its relevant characteristics, so called "properties". The purpose of developing this TReAC model is to assist the elicitation of trust requirements at the early stage of the development of the autonomous car.

Based on the literature review conducted, we found that each of the trust attributes is associated with at least one property that is related to the usage of autonomous car. Figure 2 show the relationship between trust attributes and trust properties. The detailed description of the attributes is explained below.

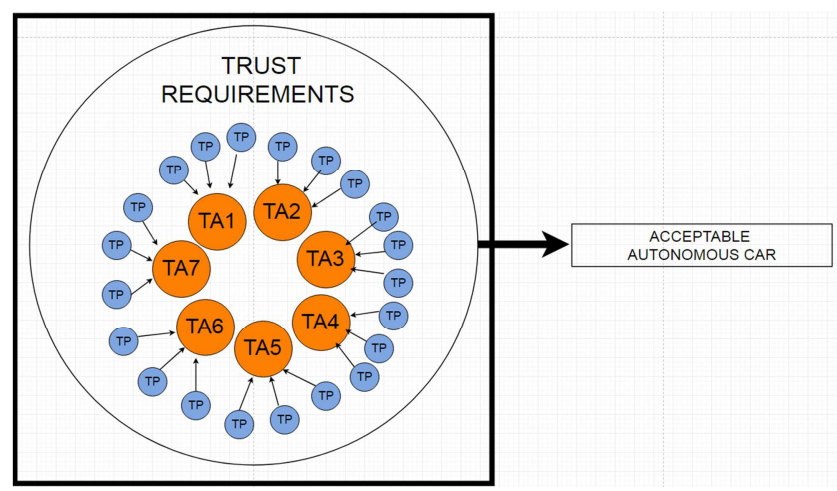

Figure 1. Trust Requirements Model in Autonomous Car (TReAC Model).

\subsection{Trust Requirements Attributes and Properties}

Figure 1 shows that there are seven (7) trust requirement attributes (TA). TA is defined as the quality attributes of a system. Trust properties (TP) refers to the characteristic of the attributes. Table 2 shows the example of trust attributes and its association with its properties. The seven attributes are:

$\begin{array}{llll}\text { I. } & \text { Security } & \text { V. } & \text { Performance } \\ \text { II. } & \text { Safety } & \text { VI. } & \text { User's Experience } \\ \text { III. } & \text { Privacy } & \text { VII. } & \text { Economic Value } \\ \text { IV. } & \text { Reliability } & & \end{array}$

\subsubsection{Security}

Security attributes is one of the main determinants for users to accept the autonomous cars. Security relates to an attribute that protects the digital information and data from any danger or threat from any malicious. Examples of the properties that relate to this attribute are data sharing, global positioning system (GPS) and visible vehicle identification number. Other types of security property in the autonomous car relates to anti-theft system and the vehicle access system such as smart key, remote keyless entry system, and remote panic features.

\subsubsection{Safety}

The second trust requirement attribute is safety. Safety relates to the ability of the autonomous car to ensure safety of its users and other people surrounding it. Examples of the properties related to safety are automatic steering, intelligent paring assist system, blind spot system, adaptive light control and camera sensor technology. The automatic steering has a safety feature as it can wake the driver when he/she is sleepy and smart cruise control is equipped with an intelligent speed adaptation, in which it slows down during heavy traffic. In this case, autonomous cars with safety attributes will have high level of acceptance in comparison to cars without safety attributes.

\subsubsection{Privacy}

Privacy is found to be another trust requirements attribute that influences the acceptance of users on autonomous cars. Examples of the properties related to privacy attributes are location of information, cloud storage, crash data retrieval, event data retrieval and cabin monitoring system. The cloud data storage allows information to be stored in cloud for 
vehicle-to-vehicle communication. Additionally, privacy attributes is closely related to security attribute. This is because automakers need to develop a high level security to avoid the privacy of user being exposed to hackers. Therefore, the security and privacy attributes need to be addressed at the same time to achieve high level of user's acceptance of the autonomous cars.

\subsubsection{Reliability}

Reliability relates to the ability of the car to perform well consistently. Reliability attribute is measured based on the rating system for head protection technology, rating system for blind spot technology, rating system for advanced safety assist technology and national highway traffic safety administration (NHTSFA). Additionally, a new car assessment for Southeast Asian countries rating (ASEAN NCAP) is also a test of reliability properties in autonomous car, especially in South East Asia. This ASEAN NCAP program is targeted to evaluate car safety standards, raise user awareness and thus encourage am market for safer car in the region. In this case, before commercializing autonomous car, automaker needs to assess the autonomous car to ensure that reliable cars are developed. Autonomous cars that receive high level of reliability influence user's acceptance.

\subsubsection{Performance}

Performance refers to the operation or the function of the autonomous car, primarily related to the effectiveness as of the engine of the autonomous car. The properties related to the performance attribute are engine smart cities, weathersensitive tires, tires sensor, active suspension control system and electronic transmission control. These features contribute to users acceptance of the autonomous cars.

\subsubsection{User's Experience}

User experience is also considered as an important trust requirement attribute for the autonomous cars. The user experience focuses on properties related to user's satisfaction when using the autonomous cars. Examples of properties relate to user's experience are the push start button, screen touch control, keyless entry, garage door system, design dashboard and on-board maps and navigation. The level of user's satisfaction based on his/her experience using the autonomous car influence the user's acceptance of the autonomous cars.

\subsubsection{Economic Value}

The final trust requirement attribute relates to the economic value of the car. This attribute represents good value for money to purchase the autonomous car based on users requirement. Examples of features that relate to economic value are trusted brand, selling price, trend, product features and car warranty. There have been limited studies on this attribute in trust requirements. However, we believe the economic value is an important attribute in trust, especially in developing autonomous car in automotive industry as it relates the user's purchasing power and preference of autonomous cars.

\subsection{Relationship between Trust Attributes and Trust Properties}

Based on figure 2, its shows the relationship of trust attributes and trust properties for developing an autonomous car. Figure 2, shows that there is one- to- many relationship between trusts attribute and trust property. This means that a trust attribute can have at least one trust property. For example as shown in table 2, safety attribute has four trust properties "automatic steering", "intelligent parking assist system", "blind spot system" and "adaptive light control".

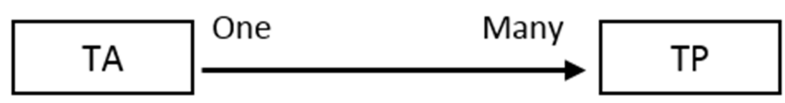

Figure 2. Relationship One TA to Many TP.

Table 2. Example of Associations between trust attributes and trust properties.

\begin{tabular}{|c|c|c|}
\hline TYPE REQUIREMENT & TRUST ATTRIBUTE (TA) & TRUST PROPERTIES (TP) \\
\hline \multirow{19}{*}{ Trust Requirements } & \multirow{5}{*}{ Safety } & Automatic steering \\
\hline & & Intelligent parking assist system \\
\hline & & Blind spot system \\
\hline & & Adaptive light control \\
\hline & & Camera sensor technology \\
\hline & \multirow{5}{*}{ Security } & Anti-Theft System \\
\hline & & Vehicle Access System \\
\hline & & Data sharing \\
\hline & & Global positioning system (GPS) \\
\hline & & Visible Vehicle Identification Number (VIN) \\
\hline & \multirow{4}{*}{ Privacy } & Location information \\
\hline & & Cloud storage \\
\hline & & Crash data retrieval \\
\hline & & Event data retrieval \\
\hline & \multirow{5}{*}{ Performance } & Engine smart cities \\
\hline & & Weather-sensitive tires \\
\hline & & Tires sensor \\
\hline & & Active suspension control system \\
\hline & & Electronic transmission control \\
\hline
\end{tabular}




\begin{tabular}{lll}
\hline TYPE REQUIREMENT & TRUST ATTRIBUTE (TA) & TRUST PROPERTIES (TP) \\
\hline & & Push Button Start \\
& Screen Touch Control \\
& Kser Experience & Keyless entry \\
& Garage door system \\
& Design Dashboard \\
& On-Board Maps And Navigation \\
& Rating System For Head Protection Technology \\
& Rating System For Blind Spot Technology \\
& Rating System Advanced Safety Assist Technologies \\
& National Highway Traffic Safety Administration (NHTSFA) \\
& Trusted Brand \\
& Selling prices \\
& Trend \\
& Product features \\
& Car warranty \\
\hline
\end{tabular}

\section{Conclusion and Future Work}

The autonomous car has brought about new trust challenges especially on user acceptance to drive that full automation car. To guarantee trust has become a key issue. In this paper, we have proposed a Trust Requirements Model for develop the user acceptance on autonomous car. This TReAC model we develop based on literature and previous study.

This study contributes on new attributes trust requirements for on user acceptance towards autonomous car the user acceptance and autonomous car, automotive industry can use this model for elicit early requirements from client or user expectation before the automotive industry develop they develop driverless car. Previous literature addressing trust often focuses on human-machine interaction with exploring trust attributes and trust on user acceptance of autonomous car. The intention was to develop trust morel to help guide the automaker in the automotive industry to elicit early requirements according user acceptance and expectation of autonomous car.

For future work, we will expand the phases of the TReAC model. We adapt this TReAC model with different domain. Then, we will validate and apply this model to a more complex in trust requirements for develop uses acceptance on the autonomous car in future.

\section{Acknowledgements}

We would like to thank Universiti Teknikal Malaysia Melaka for its support. We also would like to thank our funding: PJP/2017/FKP-CACT/S01568 and Zamalah UTeM for sponsoring the author.

\section{References}

[1] NHSTSA, "Automated Vehicle for safety," 2016. [Online]. Available: https://www.nhtsa.gov/technologyinnovation/automated-vehicles-safety.

[2] G. M. Fitch, D. S. Bowman, and R. E. Llaneras, "Human Factors: The Journal of the Human Factors and Ergonomics
Society," 2014.

[3] M. A. Nees, "Acceptance of Self-driving Cars," Proc. Hum. Factors Ergon. Soc. Annu. Meet., vol. 60, no. 1, pp. 1449$1453,2016$.

[4] C. Basu and M. Singhal, "Trust Dynamics in Human Autonomous Vehicle Interaction : A Review of Trust Models," 2016 AAAI Spring Symp. Ser., pp. 85-91, 2016.

[5] S. Robertson, "Mastering the Requirements Process Second Edition," Work, p. 15, 2006.

[6] M. Cunningham and M. A. Regan, "Autonomous Vehicles: Human Factors Issues and Future Research," Australas. Road Saf. Conf., 2015.

[7] H. Abraham, C. Lee, S. Brady, C. Fitzgerald, B. Mehler, B. Reimer, and J. F. Coughlin, "Autonomous Vehicles, Trust, and Driving Alternatives: A survey of consumer preferences," Massachusetts Inst. Technol. AgeLab, no. May, pp. 1-16, 2016.

[8] E. Fredrick, J. Mikael, and S. Jana, "Creating Appropriate Trust for Autonomous Vehicle Systems: A Framework for Human-Machine Interaction Design," 95th Annu. Meet. Transp. Res. Board, vol. 48, no. 1, pp. 1-7, 2017.

[9] D.-H. S. Jae-Gil Lee, Ki Joon Kim, Sangwon Lee and Sangwon Lee, "Can Autonomous Vehicles Be Safe and Trustworthy? Effects of Appearance and Autonomy of Unmanned Driving Systems," Int. J. Hum. Comput. Interact., 2015.

[10] I. Pettersson and I. C. M. Karlsson, "Setting the stage for autonomous cars: a pilot study of future autonomous driving experiences.," IET Intell. Transp. Syst., vol. 9, no. 7, pp. 694$701,2015$.

[11] G. Khurana, S., Chandna, A., \& Batra, "How much human do we need in a car? The evolution of artificial intelligence and the acceptance of autonomous vehicles.," EYGM Limited., 2016.

[12] H. Reese, "Updated: Autonomous driving levels 0 to 5: Understanding the differences The National Highway Traffic Safety Administration adopted the Society of Automotive Engineers' levels for automated driving systems, ranging from complete driver control to full aut," techrepublic, 2016. [Online]. Available: https://www.techrepublic.com/article/autonomous-drivinglevels-0-to-5-understanding-the-differences/. 
[13] \& Flemisch, F., Kaussner, A., Petermann, I., Schieben, A. and N. Schöming, "HAVE-IT. Highly automated vehicles for intelligent transport. Validation of concept on optimum task repartition," Regensburg, Ger. Cont. Automot. Gmb H., 2011.

[14] G. H. Walker, N. A. Stanton, and M. S. Young, "Where Is Computing Driving Cars?," vol. 13, no. 2, pp. 203-229, 2001.
[15] N. A. Stanton, M. S. Young, N. A. Stanton, M. S. Young, and H. Guy, "The psychology of driving automation : A discussion with Professor Don Norman," no. January, 2016. 